\title{
Bifurcation analysis of a three-species ecological system with time delay and harvesting
}

Zizhen Zhang ${ }^{1}$ and Aying Wan ${ }^{2 *}$

\section{"Correspondence:}

wanaying1@aliyun.com

${ }^{2} \mathrm{~S} c h o o l$ of Mathematics and

Statistics, Hulunbuir College,

Hulunbuir, 021008, China

Full list of author information is

available at the end of the article

\section{Springer}

\begin{abstract}
This paper deals with a three-species ecological system with time delay and harvesting. Sufficient conditions guaranteeing the local stability and the occurrence of Hopf bifurcation for the system are obtained. Further, the properties of Hopf bifurcation are investigated using the center manifold theorem and normal form theory. Computer simulations are carried out to illustrate the theoretical predictions. Finally, biological meaning and a conclusion are presented.
\end{abstract}

MSC: 34C15; 34C23; 37G15; 37N25

Keywords: delay; Hopf bifurcation; ecological system; periodic solution

\section{Introduction}

Predator-prey interaction has always been an important issue in mathematical modeling of ecological processes [1]. In particular, there is a lot of literature on two-species predator-prey systems [2-8]. In nature, however, there is often the interaction among multiple species, whose relationships are more complex than those in two species. Therefore, it is more realistic to investigate multiple-species predator-prey systems. Based on this, Upadhyay and Tiwari [9] proposed the following food chain system that describes the interaction among phytoplankton, zooplankton and fish:

$$
\left\{\begin{array}{l}
\frac{d P(t)}{d t}=a P(t)-b P^{2}(t)-\frac{\varepsilon_{1} P(t) Z(t)}{P(t)+d}, \\
\frac{d Z(t)}{d t}=\frac{\varepsilon_{2} P(t) Z(t)}{P(t)+d}-\gamma Z(t)-\frac{\varepsilon_{3} Z^{2}(t) F(t)}{Z^{2}(t)+\eta^{2}}, \\
\frac{d F(t)}{d t}=\frac{\varepsilon_{4} Z^{2}(t) F(t)}{Z^{2}(t)+\eta^{2}}-\delta_{1} F(t)-\delta_{2} F^{2}(t)-q E F(t),
\end{array}\right.
$$

where $P(t), Z(t)$ and $F(t)$ are the densities of phytoplankton, zooplankton and fish at time $t$, respectively. $a$ is the growth rate of phytoplankton; $b$ is the intraspecific competition rate of phytoplankton; $\frac{\varepsilon_{1} P(t) Z(t)}{P(t)+d}$ is the response function of zooplankton, $\varepsilon_{1}$ is the capturing rate of zooplankton, $\varepsilon_{2} / \varepsilon_{1}$ is the rate of conversing phytoplankton into new zooplankton, $d$ is the half-saturation constant of the phytoplankton density; $\frac{\varepsilon_{3} Z^{2}(t) F(t)}{Z^{2}(t)+\eta^{2}}$ is the response function of fish, $\varepsilon_{3}$ is the capturing rate of fish, $\varepsilon_{4} / \varepsilon_{3}$ is the rate of conversing zooplankton into new fish, $\eta$ is the half-saturation constant of the zooplankton density; $\gamma$ is the mortality rate of zooplankton; $\delta_{1}$ is the mortality rate of fish; $\delta_{2}$ is the intraspecific competition rate of

(c) The Author(s) 2017. This article is distributed under the terms of the Creative Commons Attribution 4.0 International License (http://creativecommons.org/licenses/by/4.0/), which permits unrestricted use, distribution, and reproduction in any medium, provided you give appropriate credit to the original author(s) and the source, provide a link to the Creative Commons license, and indicate if changes were made. 
fish; $q$ is the catchability coefficient and $E$ is catch per unit effort. Upadhyay and Tiwari [9] studied the stability of system (1) and discussed optimal harvesting policy.

Obviously, Upadhyay and Tiwari [9] neglected the time delay due to gestation of zooplankton and fish. The consumption of phytoplankton by zooplankton throughout its past history governs the present birth rate of zooplankton. Likewise, the consumption of zooplankton by fish throughout its past history governs the present birth rate of fish. Time delay can play an important role in the dynamics of a predator-prey system. It can cause a stable equilibrium to become unstable and cause the population to fluctuate [10-16]. Therefore, it is more realistic to take into account the effect of the time delay due to gestation of zooplankton and fish. Inspired by this idea, we consider the following predator-prey system with time delay:

$$
\left\{\begin{array}{l}
\frac{d P(t)}{d t}=a P(t)-b P^{2}(t)-\frac{\varepsilon_{1} P(t) Z(t)}{P(t)+d} \\
\frac{d Z(t)}{d t}=\frac{\varepsilon_{2} P(t-\tau) Z(t-\tau)}{P(t-\tau)+d}-\gamma Z(t)-\frac{\varepsilon_{3} Z^{2}(t) F(t)}{Z^{2}(t)+\eta^{2}}, \\
\frac{d F(t)}{d t}=\frac{\varepsilon_{4} Z^{2}(t-\tau) F(t-\tau)}{Z^{2}(t-\tau)+\eta^{2}}-\delta_{1} F(t)-\delta_{2} F^{2}(t)-q E F(t),
\end{array}\right.
$$

where $\tau$ is the time delay due to the gestation of zooplankton and fish.

This paper is organized as follows. In the next section, we analyze the existence of the Hopf bifurcation. Then, we investigate the direction of the Hopf bifurcation and the stability of bifurcating periodic solutions in Section 3. In Section 4, we give some numerical simulations to demonstrate the theoretical results obtained in this paper. Our conclusion is drawn in the final section.

\section{Existence of the Hopf bifurcation}

Based on the analysis in [9] and by direct computation, we can conclude that if the condition $\left(H_{0}\right): a>b P_{*} \frac{\varepsilon_{4} Z_{*}^{2}}{Z_{*}^{2}+\eta^{2}}>\delta_{1}+q E$ is satisfied, then system (2) has a unique positive equilibrium $E_{*}\left(P_{*}, Z_{*}, F_{*}\right)$, where

$$
\begin{aligned}
& Z_{*}=\frac{\left(a-b P_{*}\right)\left(P_{*}+d\right)}{\varepsilon_{1}}, \\
& F_{*}=\frac{\varepsilon_{4} Z_{*}^{2}}{\delta_{2}\left(Z_{*}^{2}+\eta^{2}\right)}-\frac{\delta_{1}+q E}{\delta_{2}},
\end{aligned}
$$

and $P_{*}$ is the positive root of the following equation:

$$
A_{9} P^{9}+A_{8} P^{8}+A_{7} P^{7}+A_{6} P^{6}+A_{5} P^{5}+A_{4} P^{4}+A_{3} P^{3}+A_{2} P^{2}+A_{1} P_{1}+A_{0}=0
$$

where

$$
\begin{aligned}
A_{0}= & a d^{2} \varepsilon_{1} \varepsilon_{3}\left(\delta_{1}+q E\right)\left(\varepsilon_{1}^{2} \eta^{2}+a^{2} d^{2}\right)+\varepsilon_{1}^{4} \eta^{4} \delta_{2}^{2} \gamma^{2} d-2 a^{2} d^{3} \delta_{2} \gamma \varepsilon_{1}^{2} \eta^{2} \\
& -a^{3} d^{4} \varepsilon_{1} \varepsilon_{3} \varepsilon_{4}-\delta_{2} \gamma a^{4} d^{5} \\
A_{1}= & \delta_{2} \varepsilon_{3} a^{4} d^{4}+\varepsilon_{1}^{4} \eta^{4} \delta_{2}\left(\varepsilon_{3}-\gamma\right)-\delta_{2} \gamma\left(5 a^{4} d^{4}-4 a^{3} b d^{5}\right)+2 a^{2} d^{2} \delta_{2} \varepsilon_{3} \varepsilon_{1}^{2} \eta^{2} \\
& -2 \delta_{2} \gamma \varepsilon_{1}^{2} \eta^{2}\left(3 a^{2} d^{2}-2 a b d^{3}\right)+\varepsilon_{1}^{3} \varepsilon_{3} \eta^{2}\left(\delta_{1}+q E\right)\left(3 a d-2 b d-b d^{2}\right) \\
& +\varepsilon_{1} \varepsilon_{3}\left(\delta_{2}+q E-\varepsilon_{4}\right)\left(4 a^{3} d^{3}-3 a^{2} b d^{4}\right),
\end{aligned}
$$




$$
\begin{aligned}
& A_{2}=3 a d^{2} \varepsilon_{1} \varepsilon_{3}\left(\delta_{2}+q E-\varepsilon_{4}\right)\left(2 a^{2}-4 a b d+b^{2} d^{2}\right)+a \varepsilon_{1}^{3} \varepsilon_{3} \eta^{2}\left(\delta_{1}+q E\right) \\
& -2 \delta_{2} \gamma \varepsilon_{1}^{2} \eta^{2}\left(3 a^{2} d-4 a b d^{2}+b^{2} d^{3}\right)+4 a d \delta_{2} \varepsilon_{3}(a-b d)\left(\varepsilon_{1}^{2} \eta^{2}+a^{2} d^{2}\right) \\
& -a^{2} d^{3} \delta_{2} \gamma\left(6 b^{2} d^{2}-20 a b d+10 a^{2}\right) \text {, } \\
& A_{3}=2 \delta_{2} \delta_{3} \varepsilon_{1}^{2} \eta^{2}\left(b^{2} d^{2}-2 a b d+a^{2}\right)-2 \delta_{2} \gamma \varepsilon_{1}^{2} \eta^{2}\left(3 b^{2} d^{2}-4 a b d+a^{2}\right) \\
& +\varepsilon_{1} \varepsilon_{3}\left(\delta_{1}+q E-\varepsilon_{4}\right)\left(4 a^{3} d-18 a^{2} b d^{2}+12 a b^{2} d^{3}-b^{3} d^{4}\right) \\
& -b \varepsilon_{1}^{3} \varepsilon_{3} \eta^{2}\left(\delta_{1}+q E\right)-a d^{2} \delta_{2} \gamma\left(30 a b^{2} d^{2}-40 a^{2} b d+10 a^{3}-4 b^{3} d^{3}\right) \\
& +a^{2} d^{2} \delta_{2} \delta_{3}\left(6 b^{2} d^{2}-16 a b d+6 a^{2}\right) \text {, } \\
& A_{4}=3 a d^{2} \varepsilon_{1} \varepsilon_{3}\left(\delta_{1}+q E-\varepsilon_{4}\right)\left(a^{3}-3 a^{2} b d+18 a b^{2} d^{2}-4 b^{3} d^{3}\right) \\
& +4 \delta_{2} \varepsilon_{3} \varepsilon_{1}^{2} \eta^{2}\left(b^{2} d-a b\right)-2 \delta_{2} \gamma \varepsilon_{1}^{2} \eta^{2}\left(3 b^{2} d-2 a b\right) \\
& -\delta_{2} \gamma\left(60 a^{2} b^{2} d^{3}-20 a b^{3} d^{4}-40 a^{3} b d^{2}+5 a^{4} d+a^{4} d^{2}+b^{4} d^{5}\right) \\
& +4 a d \delta_{2} \varepsilon_{3}\left(6 a b d^{2}-b^{3} d^{3}-6 a^{2} b d+a^{3}\right), \\
& A_{5}=\delta_{2} \varepsilon_{3}\left(b^{4} d^{4}-16 a b^{3} d^{3}+36 a^{2} b^{2} d^{2}-16 a^{3} b d+a^{4}+a^{4} d\right) \\
& +2 b^{2} \varepsilon_{3} \varepsilon_{1}^{2} \eta^{2}\left(\delta_{2}-\gamma\right)+3 b \varepsilon_{1} \varepsilon_{3}\left(\delta_{1}+q E-\varepsilon_{4}\right)\left(4 a b d-2 b^{2} d^{2}-a^{2}\right) \\
& -\delta_{2} \gamma\left(10 b^{4} d^{4}+60 a^{2} b^{2} d^{2}-40 a b^{3} d^{3}-20 a^{3} b d+a^{4} d+a^{4}\right) \text {, } \\
& A_{6}=b^{2} \varepsilon_{1} \varepsilon_{3}\left(\delta_{1}+q E-\varepsilon_{4}\right)(3 a-4 b d)+4 b \delta_{2} \varepsilon_{3}\left(b^{3} d^{3}-6 a b^{2} d^{2}+6 a^{2} b d-a^{2}\right) \\
& -b \delta_{2} \gamma\left(10 b^{3} d^{3}-40 a b^{2} d^{2}+30 a^{2} b d-4 a^{3}\right), \\
& A_{7}=b^{2} \delta_{2} \varepsilon_{3}\left(6 b^{2} d^{2}-16 a b d+6 a^{2}\right)-b^{3} \varepsilon_{1} \varepsilon_{3}\left(\delta_{1}+q E-\varepsilon_{4}\right) \\
& -b^{2} \delta_{2} \gamma\left(10 b^{2} d^{2}-20 a b d+6 a^{2}\right) \text {, } \\
& A_{8}=4 b^{3} \delta_{2} \varepsilon_{3}(b d-a)-b^{3} \delta_{2} \gamma(5 b d-4 a), \quad A_{9}=b^{4} \delta_{2}\left(\varepsilon_{3}-\gamma\right) \text {. }
\end{aligned}
$$

Let $p(t)=P(t)-P_{*}, z(t)=Z(t)-Z_{*}, f(t)=F(t)-F_{*}$, and we still denote $p(t), z(t)$ and $f(t)$ as $P(t), Z(t)$ and $F(t)$, respectively. Then the linearized system of system $(2)$ at $E_{*}\left(P_{*}, Z_{*}, F_{*}\right)$ is given by

$$
\frac{d}{d t}\left(\begin{array}{l}
P(t) \\
Z(t) \\
F(t)
\end{array}\right)=\left(\begin{array}{ccc}
a_{1} & a_{2} & 0 \\
0 & a_{3} & a_{4} \\
0 & 0 & a_{5}
\end{array}\right)\left(\begin{array}{c}
P(t) \\
Z(t) \\
F(t)
\end{array}\right)+\left(\begin{array}{ccc}
0 & 0 & 0 \\
b_{1} & b_{2} & 0 \\
0 & b_{3} & b_{4}
\end{array}\right)\left(\begin{array}{c}
P(t-\tau) \\
Z(t-\tau) \\
F(t-\tau)
\end{array}\right)
$$

with

$$
\begin{aligned}
& a_{1}=a-2 b P_{*}-\frac{d \varepsilon_{1} Z_{*}}{\left(P_{*}+d\right)^{2}}, \quad a_{2}=-\frac{\varepsilon_{1} P_{*}}{P_{*}+d}, \quad a_{3}=-\gamma-\frac{2 \varepsilon_{3} \eta^{2} Z_{*} F_{*}}{\left(Z_{*}^{2}+\eta^{2}\right)^{2}}, \\
& a_{4}=-\frac{\varepsilon_{3} Z_{*}^{2}}{Z_{*}^{2}+\eta^{2}}, \quad a_{5}=-\left(\delta_{1}+q E+2 \delta_{2} F_{*}\right), \\
& b_{1}=\frac{d \varepsilon_{2} Z_{*}}{\left(P_{*}+d\right)^{2}}, \quad b_{2}=\frac{\varepsilon_{2} P_{*}}{P_{*}+d}, \quad b_{3}=\frac{2 \varepsilon_{4} \eta^{2} Z_{*} F_{*}}{\left(Z_{*}^{2}+\eta^{2}\right)^{2}}, \quad b_{4}=\frac{\varepsilon_{4} Z_{*}}{Z_{*}^{2}+\eta^{2}} .
\end{aligned}
$$


The characteristic equation of system (4) is

$$
\lambda^{3}+s_{02} \lambda^{2}+s_{01} \lambda+s_{00}+\left(s_{12} \lambda^{2}+s_{11} \lambda+s_{10}\right) e^{-\lambda \tau}+\left(s_{21} \lambda+s_{20}\right) e^{-2 \lambda \tau}=0,
$$

where

$$
\begin{aligned}
& s_{00}=-a_{1} a_{3} a_{5}, \quad s_{01}=a_{1} a_{3}+a_{1} a_{5}+a_{3} a_{5}, \\
& s_{02}=-\left(a_{1}+a_{3}+a_{5}\right), \\
& s_{10}=a_{1} a_{4} b_{3}+a_{2} a_{5} b_{1}-a_{1} a_{5} b_{2}-a_{1} a_{3} b_{4}, \\
& s_{11}=b_{2}\left(a_{1}+a_{5}\right)+b_{4}\left(a_{1}+a_{3}\right)-a_{2} b_{1}-a_{4} b_{4}, \\
& s_{12}=-\left(b_{2}+b_{4}\right), \\
& s_{20}=a_{2} b_{1} b_{4}-a_{1} b_{2} b_{4}, \quad s_{21}=b_{2} b_{4} .
\end{aligned}
$$

Multiplying by $e^{\lambda \tau}$, Eq. (5) becomes

$$
s_{12} \lambda^{2}+s_{11} \lambda+s_{10}+\left(\lambda^{3}+s_{02} \lambda^{2}+s_{01} \lambda+s_{00}\right) e^{\lambda \tau}+\left(s_{21} \lambda+s_{20}\right) e^{-\lambda \tau}=0 .
$$

When $\tau=0$, Eq. (6) becomes

$$
\lambda^{3}+\left(s_{02}+s_{12}\right) \lambda^{2}+\left(s_{01}+s_{11}+s_{21}\right) \lambda+s_{00}+s_{10}+s_{20}=0 .
$$

Thus, by the Routh-Hurwitz criterion, $E_{*}\left(P_{*}, Z_{*}, F_{*}\right)$ is asymptotically stable if the condition $\left(H_{1}\right): s_{02}+s_{12}>0, s_{01}+s_{11}+s_{21}>0, s_{00}+s_{10}+s_{20}>0$ and $\left(s_{02}+s_{12}\right)\left(s_{01}+s_{11}+s_{21}\right)>$ $s_{00}+s_{10}+s_{20}$ holds.

For $\tau>0$, let $\lambda=i \omega(\omega>0)$ be the root of Eq. (6), then

$$
\left\{\begin{array}{l}
\left(s_{00}+s_{20}-s_{02} \omega^{2}\right) \omega \cos \tau \omega+\left(\left(s_{21}-r_{01}\right) \omega+\omega^{3}\right) \sin \tau \omega=s_{12} \omega^{2}-s_{10} \\
\left(s_{00}-s_{20}-s_{02} \omega^{2}\right) \omega \sin \tau \omega+\left(\left(s_{21}+r_{01}\right) \omega+\omega^{3}\right) \sin \tau \omega=-s_{11} \omega
\end{array}\right.
$$

Thus, we can obtain

$$
\cos \tau \omega=\frac{g_{1}(\omega)}{g_{0}(\omega)}, \quad \sin \tau \omega=\frac{g_{2}(\omega)}{g_{0}(\omega)},
$$

where

$$
\begin{aligned}
g_{0}(\omega)= & \omega^{6}+\left(s_{02}^{2}+2 s_{01}\right) \omega^{4} \\
& +\left(s_{21}^{2}-s_{01}^{2}-2 s_{00} s_{02}\right) \omega^{2}+s_{00}^{2}-s_{20}^{2}, \\
g_{1}(\omega)= & \left(s_{11}+s_{02} s_{12}\right) \omega^{4} \\
& +\left[s_{11}\left(s_{21}-s_{01}\right)-s_{12}\left(s_{00}-s_{20}\right)+s_{10} s_{02}\right] \omega^{2}-s_{10}\left(s_{00}-s_{20}\right), \\
g_{2}(\omega)= & s_{12} \omega^{5}+\left[s_{02} s_{11}-s_{10}-s_{12}\left(s_{21}+s_{01}\right)\right] \omega^{3} \\
& +\left[s_{10}\left(s_{21}+s_{01}\right)-s_{11}\left(s_{00}+s_{20}\right)\right] \omega .
\end{aligned}
$$


It follows that

$$
g_{0}^{2}(\omega)-g_{1}^{2}(\omega)-g_{2}^{2}(\omega)=0
$$

In order to obtain the main results in this paper, we suppose that $\left(H_{2}\right)$ : Eq. (9) has at least one positive root $\omega_{0}$. Thus, Eq. (6) has a pair of purely imaginary roots $\pm i \omega_{0}$.

$$
\tau_{0}= \begin{cases}\frac{1}{\omega_{0}} \times \arccos \left\{\frac{g_{1}\left(\omega_{0}\right)}{g_{0}\left(\omega_{0}\right)}\right\}, & \sin \left(\omega_{0} \tau_{0}\right) \geq 0, \\ \frac{1}{\omega_{0}} \times\left(2 \pi-\arccos \left\{\frac{g_{1}\left(\omega_{0}\right)}{g_{0}\left(\omega_{0}\right)}\right\}\right), & \sin \left(\omega_{0} \tau_{0}\right)<0 .\end{cases}
$$

Differentiating Eq. (6) with respect to $\tau$, it follows that

$$
\left[\frac{d \lambda}{d \tau}\right]^{-1}=\frac{2 s_{12} \lambda+s_{11}+\left(3 \lambda^{2}+2 s_{02} \lambda+s_{01}\right) e^{\lambda \tau}+s_{21} e^{-\lambda \tau}}{\lambda\left[\left(s_{21} \lambda+s_{20}\right) e^{-\lambda \tau}-\left(\lambda^{3}+s_{02} \lambda^{2}+s_{01} \lambda+s_{00}\right) e^{\lambda \tau}\right]}-\frac{\tau}{\lambda} .
$$

Further, we have

$$
\operatorname{Re}\left[\frac{d \lambda}{d \tau}\right]_{\tau=\tau_{0}}^{-1}=\frac{U_{1} V_{1}+U_{2} V_{2}}{V_{1}^{2}+V_{2}^{2}}
$$

where

$$
\begin{aligned}
& U_{1}=\left(s_{01}+s_{21}-3 \omega_{0}^{2}\right) \cos \tau_{0} \omega_{0}-2 s_{02} \omega_{0} \sin \tau_{0} \omega_{0}+s_{11}, \\
& U_{2}=\left(s_{01}-s_{21}-3 \omega_{0}^{2}\right) \cos \tau_{0} \omega_{0}+2 s_{02} \omega_{0} \sin \tau_{0} \omega_{0}+s_{12} \omega_{0}, \\
& V_{1}=\left[\left(s_{20}+s_{00}\right) \omega_{0}-s_{02} \omega_{0}^{2}\right] \sin \tau_{0} \omega_{0}-\left[\left(s_{21}-s_{01}\right) \omega_{0}^{2}+\omega_{0}^{4}\right] \cos \tau_{0} \omega_{0}, \\
& V_{2}=\left[\left(s_{20}-s_{00}\right) \omega_{0}+s_{02} \omega_{0}^{2}\right] \cos \tau_{0} \omega_{0}+\left[\left(s_{21}+s_{01}\right) \omega_{0}^{2}-\omega_{0}^{4}\right] \sin \tau_{0} \omega_{0} .
\end{aligned}
$$

Hence the transversality condition is satisfied if the condition $\left(H_{3}\right): U_{1} U_{2}+V_{1} V_{2} \neq 0$ holds. Then we have the following according to the Hopf bifurcation theorem in [17].

Theorem 1 For system (2), if conditions $\left(H_{0}\right)-\left(H_{3}\right)$ hold, $E_{*}\left(P_{*}, Z_{*}, F_{*}\right)$ is asymptotically stable for $\tau \in\left[0, \tau_{0}\right)$ and system (2) undergoes a Hopf bifurcation at $E_{*}\left(P_{*}, Z_{*}, F_{*}\right)$ when $\tau=\tau_{0}$.

\section{Stability and direction of the Hopf bifurcation}

We already know that system (2) will undergo a Hopf bifurcation at $E_{*}\left(P_{*}, Z_{*}, F_{*}\right)$ when the time delay $\tau$ passes through the critical value $\tau_{0}$. We investigate the direction, stability and period of the Hopf bifurcation by means of the techniques introduced in [17]. Let $\tau=\tau_{0}+\mu, \mu \in R$. Then $\mu=0$ is the Hopf bifurcation value of the system.

Define the space of continuous real-valued functions as $C=C\left([-1,0], R^{3}\right)$. Let $u_{1}(t)=$ $P(t)-P_{*}, u_{2}(t)=Z(t)-Z_{*}, u_{3}(t)=F(t)-F_{*}$ and $u_{i}(t)=u_{i}(\tau t)$ for $i=1,2,3$. System (2) then transforms to the following form:

$$
\dot{u}(t)=L_{\mu} u_{t}+F\left(\mu, u_{t}\right),
$$

where $u_{t}=\left(u_{1}(t), u_{2}(t), u_{3}(t)\right)^{T} \in C=C\left([-1,0], R^{3}\right)$,

$$
L_{\mu} \phi=\left(\tau_{0}+\mu\right)(M \phi(0)+N \phi(-1))
$$


and

$$
F(\mu, \phi)=\left(\tau_{0}+\mu\right)\left(F_{1}, F_{2}, F_{3}\right)^{T},
$$

where

$$
M=\left(\begin{array}{ccc}
a_{1} & a_{2} & 0 \\
0 & a_{3} & a_{4} \\
0 & 0 & a_{5}
\end{array}\right), \quad N=\left(\begin{array}{ccc}
0 & 0 & 0 \\
b_{1} & b_{2} & 0 \\
0 & b_{3} & b_{4}
\end{array}\right)
$$

and

$$
\begin{aligned}
F_{1}= & a_{11} \phi_{1}^{2}(0)+a_{12} \phi_{1}(0) \phi_{2}(0)+a_{13} \phi_{1}^{2}(0) \phi_{2}(0)+a_{14} \phi_{1}^{3}(0)+\cdots, \\
F_{2}= & a_{21} \phi_{2}^{2}(0)+a_{22} \phi_{2}(0) \phi_{3}(0)+a_{23} \phi_{2}^{2}(0) \phi_{3}(0)+a_{24} \phi_{2}^{3}(0) \\
& +a_{25} \phi_{1}^{2}(-1)+a_{26} \phi_{1}(-1) \phi_{2}(-1)+a_{27} \phi_{1}^{2}(-1) \phi_{2}(-1)+a_{28} \phi_{1}^{3}(-1)+\cdots, \\
& { }_{F_{3}=} a_{31} \phi_{3}^{2}(0)+a_{32} \phi_{2}^{2}(-1)+a_{33} \phi_{2}(-1) \phi_{3}(-1)+a_{34} \phi_{2}^{2}(-1) \phi_{3}(-1)+a_{35} \phi_{2}^{3}(-1)+\cdots,
\end{aligned}
$$

with

$$
\begin{aligned}
& a_{11}=-b+\frac{d \varepsilon_{1} Z_{*}}{\left(P_{*}+d\right)^{3}}, \quad a_{12}=\frac{d \varepsilon_{1}}{\left(P_{*}+d\right)^{2}}, \\
& a_{13}=\frac{d \varepsilon_{1}}{\left(P_{*}+d\right)^{3}}, \quad a_{14}=-\frac{d \varepsilon_{1} Z_{*}}{\left(P_{*}+d\right)^{4}}, \\
& a_{21}=\frac{\varepsilon_{3} \eta^{2} F_{*}\left(3 Z_{*}^{2}-\eta^{2}\right)}{\left(Z_{*}^{2}+\eta^{2}\right)^{3}}, \quad a_{22}=-\frac{\varepsilon_{3} \eta^{2} Z_{*}}{\left(Z_{*}^{2}+\eta^{2}\right)^{2}}, \\
& a_{23}=\frac{\varepsilon_{3} \eta^{2}\left(3 Z_{*}^{2}-\eta^{2}\right)}{\left(Z_{*}^{2}+\eta^{2}\right)^{3}}, \quad a_{24}=\frac{6 \varepsilon_{3} \eta^{2} F_{*}\left(\eta^{2}-Z_{*}^{2}\right)}{\left(Z_{*}^{2}+\eta^{2}\right)^{4}}, \\
& a_{25}=-\frac{d \varepsilon_{2} Z_{*}}{\left(P_{*}+d\right)^{3}}, \quad a_{26}=\frac{d \varepsilon_{2}}{\left(P_{*}+d\right)^{2}}, \\
& a_{27}=-\frac{d \varepsilon_{2}}{\left(P_{*}+d\right)^{3}}, \quad a_{28}=\frac{d \varepsilon_{2} Z_{*}}{\left(P_{*}+d\right)^{4}}, \\
& a_{31}=-\delta_{2}, \quad a_{32}=-\frac{\varepsilon_{4} \eta^{2} F_{*}\left(3 Z_{*}^{2}-\eta^{2}\right)}{\left(Z_{*}^{2}+\eta^{2}\right)^{3}}, \quad a_{33}=\frac{\varepsilon_{4} \eta^{2} Z_{*}}{\left(Z_{*}^{2}+\eta^{2}\right)^{2}}, \\
& a_{34}=-\frac{\varepsilon_{4} \eta^{2}\left(3 Z_{*}^{2}-\eta^{2}\right)}{\left(Z_{*}^{2}+\eta^{2}\right)^{3}}, \quad a_{35}=-\frac{6 \varepsilon_{3} \eta^{2} F_{*}\left(\eta^{2}-Z_{*}^{2}\right)}{\left(Z_{*}^{2}+\eta^{2}\right)^{4}} .
\end{aligned}
$$

By the representation theorem, there exists a $3 \times 3$ matrix function $\eta(\theta, \mu), \theta \in[-1,0]$ such that

$$
L_{\mu} \phi=\int_{-1}^{0} d \eta(\theta, \mu) \phi(\theta), \quad \phi \in C .
$$

In view of Eq. (12), we can choose

$$
\eta(\theta, \mu)=\left(\tau_{0}+\mu\right)(M \delta(\theta)+N \delta(\theta+1))
$$

where $\delta$ is the Dirac delta function. 
For $\phi \in C\left([-1,0], R^{3}\right)$, define

$$
A(\mu) \phi= \begin{cases}\frac{d \phi(\theta)}{d \theta}, & -1 \leq \theta<0 \\ \int_{-1}^{0} d \eta(\theta, \mu) \phi(\theta), & \theta=0\end{cases}
$$

and

$$
R(\mu) \phi= \begin{cases}0, & -1 \leq \theta<0, \\ F(\mu, \phi), & \theta=0 .\end{cases}
$$

Then system (11) is equivalent to

$$
\dot{u}(t)=A(\mu) u_{t}+R(\mu) u_{t},
$$

where $u_{t}(\theta)=u(t+\theta)$ for $\theta \in[-1,0]$.

For $\varphi \in C^{1}\left([0,1],\left(R^{3}\right)^{*}\right)$, define

$$
A^{*}(\varphi)= \begin{cases}-\frac{d \varphi(s)}{d s}, & 0<s \leq 1, \\ \int_{-1}^{0} d \eta^{T}(s, 0) \varphi(-s), & s=0,\end{cases}
$$

and a bilinear inner product

$$
\langle\varphi(s), \phi(\theta)\rangle=\bar{\varphi}(0) \phi(0)-\int_{\theta=-1}^{0} \int_{\xi=0}^{\theta} \bar{\varphi}(\xi-\theta) d \eta(\theta) \phi(\xi) d \xi
$$

where $\eta(\theta)=\eta(\theta, 0)$. Then $A(0)$ and $A^{*}$ are adjoint operators.

Suppose that $\rho(\theta)=\left(1, \rho_{2}, \rho_{3}\right)^{T} e^{i \omega_{0} \tau_{0} \theta}$ is the eigenvector of $A(0)$ belonging to $+i \omega_{0} \tau_{0}$ and $\rho^{*}(s)=D\left(1, \rho_{2}^{*}, \rho_{3}^{*}\right) e^{i \omega_{0} \tau_{0} s}$ is the eigenvector of $A^{*}(0)$ belonging to $-i \omega_{0} \tau_{0}$. By a direct computation, we obtain

$$
\begin{aligned}
& \rho_{2}=\frac{i \omega_{0}-a_{1}}{a_{2}}, \quad \rho_{3}=\frac{a_{4}\left(i \omega_{0}+a_{1}\right) e^{-i \tau_{0} \omega_{0}}}{b_{1}\left(i \omega_{0}-a_{5}-b_{4} e^{-i \tau_{0} \omega_{0}}\right) a_{2}}, \\
& \rho_{2}^{*}=-\frac{i \omega_{0}+a_{1}}{b_{1} e^{i \tau_{0} \omega_{0}}}, \quad \rho_{3}^{*}=\frac{a_{4}\left(i \omega_{0}+a_{1}\right) e^{-i \tau_{0} \omega_{0}}}{b_{1}\left(i \omega_{0}+a_{5}+b_{4} e^{i \tau_{0} \omega_{0}}\right)} .
\end{aligned}
$$

From Eq. (15), we can get

$$
\bar{D}=\left[1+\rho_{2} \bar{\rho}_{2}^{*}+\rho_{3} \bar{\rho}_{3}^{*}+\tau_{0} e^{-i \tau_{0} \omega_{0}}\left(b_{1} \bar{\rho}_{2}^{*}+\rho_{2}\left(b_{2} \bar{\rho}_{2}^{*}+b_{3} \bar{\rho}_{3}^{*}\right)+b_{4} \rho_{3} \bar{\rho}_{3}^{*}\right)\right]^{-1}
$$

such that $\left\langle\rho^{*}, \rho\right\rangle=1$.

Next, we can obtain the coefficients by using the method introduced in [17] and a computation process similar to that in $[2,18-21]$ :

$$
\begin{aligned}
g_{20}= & 2 \tau_{0} \bar{D}\left[a_{11}+a_{12} \rho_{2}+\bar{\rho}_{2}^{*}\left(a_{21} \rho_{2}^{2}+a_{22} \rho_{2} \rho_{3}+a_{25} e^{-2 i \tau_{0} \omega_{0}}+a_{26} \rho_{2} e^{-2 i \tau_{0} \omega_{0}}\right)\right. \\
& \left.+\bar{\rho}_{3}^{*}\left(a_{31} \rho_{3}^{2}+a_{32} \rho_{2} e^{-2 i \tau_{0} \omega_{0}}+a_{33} \rho_{2} \rho_{3} e^{-2 i \tau_{0} \omega_{0}}\right)\right], \\
g_{11}= & \tau_{0} \bar{D}\left[2 a_{11}+a_{12}\left(\rho_{2}+\bar{\rho}_{2}\right)+\bar{\rho}_{2}^{*}\left(2 a_{21} \rho_{2} \bar{\rho}_{2}+a_{22}\left(\rho_{2} \bar{\rho}_{3}+\bar{\rho}_{2} \rho_{3}\right)+2 a_{25}\right.\right. \\
& \left.\left.+a_{26}\left(\rho_{2}+\bar{\rho}_{2}\right)\right)+\bar{\rho}_{3}^{*}\left(2 a_{31} \rho_{3} \bar{\rho}_{3}+2 a_{32} \rho_{2} \bar{\rho}_{2}+a_{33}\left(\rho_{2} \bar{\rho}_{3}+\bar{\rho}_{2} \rho_{3}\right)\right)\right],
\end{aligned}
$$




$$
\begin{aligned}
g_{02}= & 2 \tau_{0} \bar{D}\left[a_{11}+a_{12} \bar{\rho}_{2}+\bar{\rho}_{2}^{*}\left(a_{21} \bar{\rho}_{2}^{2}+a_{22} \bar{\rho}_{2} \bar{\rho}_{3}+a_{25} e^{2 i \tau_{0} \omega_{0}}+a_{26} \bar{\rho}_{2} e^{2 i \tau_{0} \omega_{0}}\right)\right. \\
& \left.+\bar{\rho}_{3}^{*}\left(a_{31} \bar{\rho}_{3}^{2}+a_{32} \bar{\rho}_{2} e^{2 i \tau_{0} \omega_{0}}+a_{33} \bar{\rho}_{2} \bar{\rho}_{3} e^{2 i \tau_{0} \omega_{0}}\right)\right] \\
g_{21}= & 2 \tau_{0} \bar{D}\left[a_{11}\left(2 W_{11}^{(1)}(0)+W_{20}^{(1)}(0)\right)+a_{12}\left(W_{11}^{(1)}(0) \rho_{2}+\frac{1}{2} W_{20}^{(1)}(0) \bar{\rho}_{2}+W_{11}^{(2)}(0)\right.\right. \\
& \left.+\frac{1}{2} W_{20}^{(2)}(0)\right)+a_{13}\left(\bar{\rho}_{2}+2 \rho_{2}\right)+3 a_{14}+\bar{\rho}_{2}^{*}\left(a_{21}\left(2 W_{11}^{(2)}(0) \rho_{2}+W_{20}^{(20}(0) \bar{\rho}_{2}\right)\right. \\
& +a_{22}\left(W_{11}^{(2)}(0) \rho_{3}+\frac{1}{2} W_{20}^{(2)}(0) \bar{\rho}_{3}+W_{11}^{(3)}(0) \rho_{2}+\frac{1}{2} W_{20}^{(3)}(0) \bar{\rho}_{2}\right) \\
& +a_{23}\left(\rho_{2}^{2} \bar{\rho}_{3}+2 \rho_{2} \bar{\rho}_{2} \rho_{3}\right)+3 a_{24} \rho_{2}^{2} \bar{\rho}_{2}+a_{25}\left(2 W_{11}^{(1)}(-1) e^{-i \tau_{0} \omega_{0}}+W_{20}^{(1)}(-1) e^{i \tau_{0} \omega_{0}}\right) \\
& +a_{26}\left(W_{11}^{(1)}(-1) \rho_{2} e^{-i \tau_{0} \omega_{0}}+\frac{1}{2} W_{20}^{(1)}(-1) \bar{\rho}_{2} e^{i \tau_{0} \omega_{0}}+W_{11}^{(2)}(-1) e^{-i \tau_{0} \omega_{0}}\right. \\
& \left.\left.+\frac{1}{2} W_{20}^{(1)}(-1) e^{i \tau_{0} \omega_{0}}\right)+a_{27}\left(\bar{\rho}_{2} e^{i \tau_{0} \omega_{0}}+2 \rho_{2} e^{-i \tau_{0} \omega_{0}}\right)+3 a_{28} e^{i \tau_{0} \omega_{0}}\right) \\
& \left.\left.+3 a_{35} \rho_{2}^{2} \bar{\rho}_{2} e^{-i \tau_{0} \omega_{0}}\right)\right], \\
& +\bar{\rho}_{3}^{*}\left(a_{31}\left(2 W_{11}^{(3)}(0) \rho_{3}+W_{20}^{(3)}(0) \bar{\rho}_{3}\right)\right. \\
& +a_{32}\left(2 W_{11}^{(2)}(-1) \rho_{2} e^{-i \tau_{0} \omega_{0}}+W_{20}^{(2)}(-1) \bar{\rho}_{2} e^{i \tau_{0} \omega_{0}}\right) \\
& +a_{33}\left(W_{11}^{(2)}(-1) \rho_{3} e^{-i \tau_{0} \omega_{0}}+\frac{1}{2} W_{20}^{(2)}(-1) \bar{\rho}_{3} e^{i \tau_{0} \omega_{0}}+W_{11}^{(3)}(-1) \rho_{2} e^{-i \tau_{0} \omega_{0}}\left(\rho_{2}^{2} \bar{\rho}_{3} e^{-i \tau_{0} \omega_{0}}+2 \rho_{2} \bar{\rho}_{2} \rho_{3} e^{-i \tau_{0} \omega_{0}}\right)\right. \\
& \left.\frac{1}{2}\right) \\
& \\
& \\
&
\end{aligned}
$$

with

$$
\begin{aligned}
& W_{20}(\theta)=\frac{i g_{20} \rho(0)}{\tau_{0} \omega_{0}} e^{i \tau_{0} \omega_{0} \theta}+\frac{i \bar{g}_{02} \bar{\rho}(0)}{3 \tau_{0} \omega_{0}} e^{-i \tau_{0} \omega_{0} \theta}+E_{1} e^{2 i \tau_{0} \omega_{0} \theta}, \\
& W_{11}(\theta)=-\frac{i g_{11} \rho(0)}{\tau_{0} \omega_{0}} e^{i \tau_{0} \omega_{0} \theta}+\frac{i \bar{g}_{11} \bar{\rho}(0)}{\tau_{0} \omega_{0}} e^{-i \tau_{0} \omega_{0} \theta}+E_{2},
\end{aligned}
$$

where $E_{1}$ and $E_{2}$ are given by the following equations, respectively:

$$
\begin{aligned}
& \left(\begin{array}{ccc}
2 i \omega_{0}-a_{1} & -a_{2} & 0 \\
-b_{1} e^{-2 i \tau_{0} \omega_{0}} & 2 i \omega_{0}-a_{3}-b_{2} e^{-2 i \tau_{0} \omega_{0}} & -a_{4} \\
0 & -b_{3} e^{-2 i \tau_{0} \omega_{0}} & 2 i \omega_{0}-a_{5}-b_{4} e^{-2 i \tau_{0} \omega_{0}}
\end{array}\right) E_{1}=2\left(\begin{array}{l}
E_{11} \\
E_{12} \\
E_{13}
\end{array}\right) \\
& \left(\begin{array}{ccc}
a_{1} & a_{2} & 0 \\
b_{1} & a_{3}+b_{2} & a_{4} \\
0 & b_{3} & a_{5}+b_{4}
\end{array}\right) E_{2}=-\left(\begin{array}{c}
E_{21} \\
E_{22} \\
E_{23}
\end{array}\right)
\end{aligned}
$$

and

$$
\begin{aligned}
& E_{11}=a_{11}+a_{12} \rho_{2}, \\
& E_{12}=a_{21} \rho_{2}^{2}+a_{22} \rho_{2} \rho_{3}+a_{25} e^{-2 i \tau_{0} \omega_{0}}+a_{26} \rho_{2} e^{-2 i \tau_{0} \omega_{0}}
\end{aligned}
$$




$$
\begin{aligned}
& E_{13}=a_{31} \rho_{3}^{2}+a_{32} \rho_{2} e^{-2 i \tau_{0} \omega_{0}}+a_{33} \rho_{2} \rho_{3} e^{-2 i \tau_{0} \omega_{0}}, \\
& E_{21}=2 a_{11}+a_{12}\left(\rho_{2}+\bar{\rho}_{2}\right), \\
& E_{22}=2 a_{21} \rho_{2} \bar{\rho}_{2}+a_{22}\left(\rho_{2} \bar{\rho}_{3}+\bar{\rho}_{2} \rho_{3}\right)+2 a_{25}+a_{26}\left(\rho_{2}+\bar{\rho}_{2}\right), \\
& E_{23}=2 a_{31} \rho_{3} \bar{\rho}_{3}+2 a_{32} \rho_{2} \bar{\rho}_{2}+a_{33}\left(\rho_{2} \bar{\rho}_{3}+\bar{\rho}_{2} \rho_{3}\right) .
\end{aligned}
$$

Then we can get the following coefficients which determine the properties of the Hopf bifurcation:

$$
\begin{aligned}
& C_{1}(0)=\frac{i}{2 \tau_{0} \omega_{0}}\left(g_{11} g_{20}-2\left|g_{11}\right|^{2}-\frac{\left|g_{02}\right|^{2}}{3}\right)+\frac{g_{21}}{2}, \\
& \mu_{2}=-\frac{\operatorname{Re}\left\{C_{1}(0)\right\}}{\operatorname{Re}\left\{\lambda^{\prime}\left(\tau_{0}\right)\right\}} \\
& \beta_{2}=2 \operatorname{Re}\left\{C_{1}(0)\right\} \\
& T_{2}=-\frac{\operatorname{Im}\left\{C_{1}(0)\right\}+\mu_{2} \operatorname{Im}\left\{\lambda^{\prime}\left(\tau_{0}\right)\right\}}{\tau_{0} \omega_{0}} .
\end{aligned}
$$

Hence, we have the following according to the results describing the properties of the Hopf bifurcation of dynamical systems in [16].

Theorem 2 For system (2), if $\mu_{2}>0\left(\mu_{2}<0\right)$, then the Hopf bifurcation is supercritical (subcritical). If $\beta_{2}<0\left(\beta_{2}>0\right)$, then the bifurcating periodic solutions are stable (unstable). If $T_{2}>0\left(T_{2}<0\right)$, then the bifurcating periodic solutions increase (decrease).

\section{Numerical example}

In this section, the dynamical behavior of system (2) is investigated numerically. We choose the following set of parameter values: $a=0.128, b=0.01, \varepsilon_{1}=0.28, d=10$, $\varepsilon_{2}=0.28, \varepsilon_{3}=0.2, \varepsilon_{4}=0.15, \eta=10, \delta_{1}=0.01, \delta_{2}=0.001, q E=0.01$. Then system (2) becomes

$$
\left\{\begin{array}{l}
\frac{d P(t)}{d t}=0.128 P(t)-0.01 P^{2}(t)-\frac{0.28 P(t) Z(t)}{P(t)+10} \\
\frac{d Z(t)}{d t}=\frac{0.28 P(t-\tau) Z(t-\tau)}{P(t-\tau)+10}-0.06 Z(t)-\frac{0.2 Z^{2}(t) F(t)}{Z^{2}(t)+100}, \\
\frac{d F(t)}{d t}=\frac{0.15 Z^{2}(t-\tau) F(t-\tau)}{Z^{2}(t-\tau)+100}-0.01 F(t)-0.001 F^{2}(t)-0.01 F(t),
\end{array}\right.
$$

from which one can obtain the unique positive equilibrium $E_{*}(4.4338,4.3127,3.5238)$ with the help of Matlab software package. Further, we have $\omega_{0}=1.0618, \tau_{0}=7.7149$, $\lambda^{\prime}\left(\tau_{0}\right)=0.5570-1.2740 i$ and $C_{1}(0)=-3.2691+1.8322 i$. From Eq. (16), we obtain $\mu_{2}=$ $5.8691>0, \beta_{2}=-6.5382<0$ and $T_{2}=0.6891>0$.

Thus, $E_{*}(4.4338,4.3127,3.5238)$ is asymptotically stable when $\tau \in\left[0, \tau_{0}\right)$. Fix $\tau=$ $5.6525<\tau_{0}$, then we can easily plot the time series of phytoplankton, zooplankton and fish and phase portrait of system (17) and find that the solution of system (17) would tend to $E_{*}(4.4338,4.3127,3.5238)$. This reveals that the densities of phytoplankton, zooplankton and fish in system (17) will tend to stabilization. This property can be illustrated by Figure 1 . When the time delay $\tau$ passes through $\tau_{0}, E_{*}(4.4338,4.3127,3.5238)$ loses its stability and a Hopf bifurcation occurs and a family of periodic solutions bifurcate from $E_{*}(4.4338,4.3127,3.5238)$. Figure 2 is plotted by fixing the time delay $\tau=9.1525>\tau_{0}$. It 

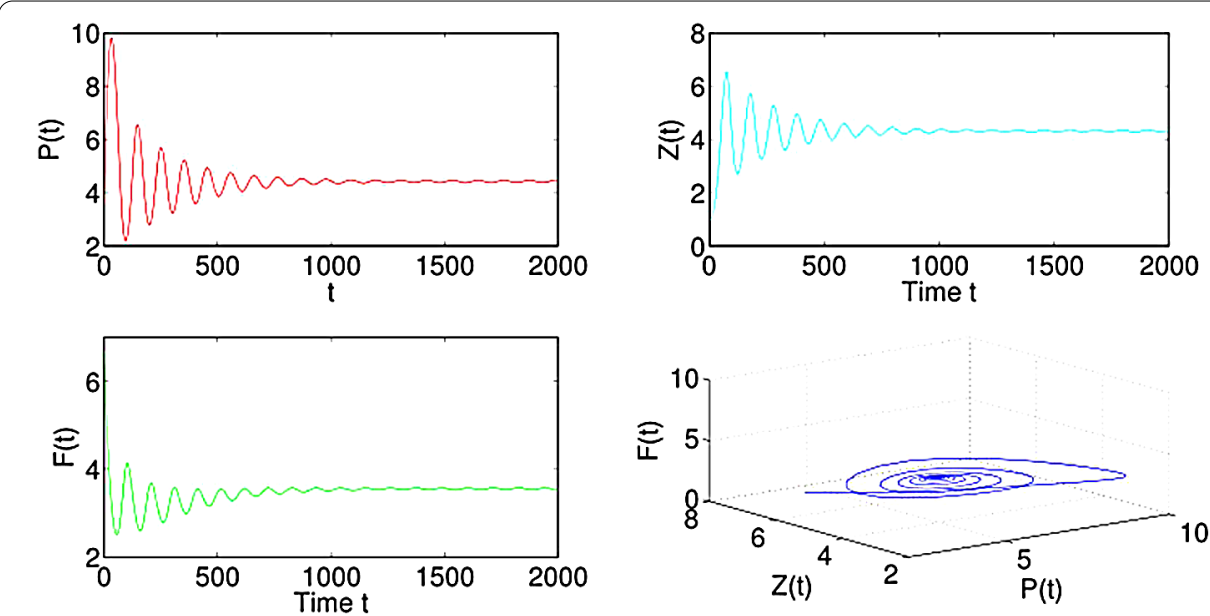

Figure $1 E_{*}$ is locally asymptotically stable when $\tau=5.6525<\tau_{0}$.
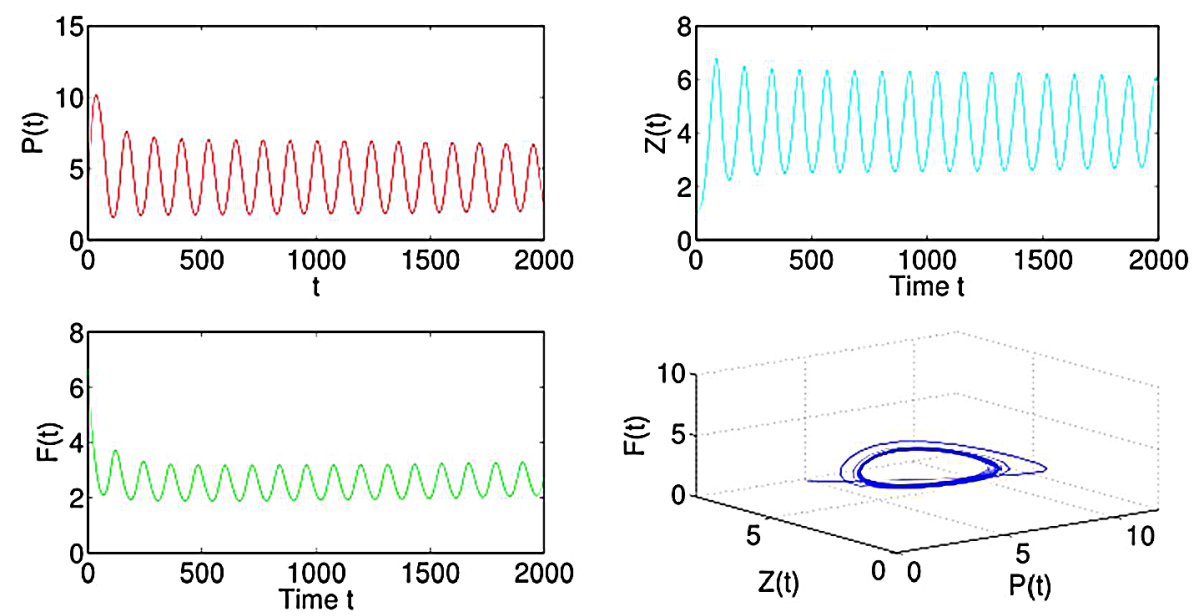

Figure 2 System (17) undergoes a Hopf bifurcation when $\tau=9.1525>\tau_{0}$.

is shown that a Hopf bifurcation occurs and a family of periodic solutions bifurcate from $E_{*}(4.4338,4.3127,3.5238)$. This reveals that the densities of phytoplankton, zooplankton and fish in system (17) will oscillate in the vicinity of $P_{*}, Z_{*}$ and $F_{*}$, respectively. Since $\mu_{2}>0, \beta_{2}<0$ and $T_{2}>0$, we know that the direction of the Hopf bifurcation at $\tau_{0}=7.7149$ is supercritical; the periodic solutions bifurcating from $E_{*}(4.4338,4.3127,3.5238)$ are stable and the period of the solutions increases.

\section{Conclusions}

In this paper, we have proposed a delayed system to study the dynamics of phytoplankton, zooplankton and fish population with Holling type II and III functional responses based on the system considered in [9]. The relationship between phytoplankton and zooplankton is described by Holling type II functional response, and the relationship between zooplankton and fish is described by Holling type III functional response. Compared with the system considered in [9], we mainly investigate the effect of time delay due to gestation of zooplankton and fish on the system. 
In the context, we have given sufficient conditions for the local stability and the existence of a Hopf bifurcation by regarding the time delay as the bifurcation parameter. We have shown that the system is asymptotically stable when the time delay is suitably small $\left(\tau \in\left[0, \tau_{0}\right)\right)$ under some certain conditions, which means that the densities of phytoplankton, zooplankton and fish in system (17) will tend to stabilization. However, the system will lose its stability once the time delay passes through the critical value $\tau_{0}$ and a Hopf bifurcation occurs, which means that the densities of phytoplankton, zooplankton and fish in the system will fluctuate in periodic oscillation form. In particular, the properties of the Hopf bifurcation have also been investigated by using the normal form theory and the center manifold theorem. Finally, a numerical example has been presented in order to verify the main results we obtained.

\section{Acknowledgements}

This work was supported by the National Natural Science Foundation of China (No. 11461024) and the Natural Science Foundation of Anhui Province (Nos 1608085QF145, 1608085QF151, 1708085MA17).

\section{Competing interests}

The authors declare that they have no competing interests.

Authors' contributions

All authors contributed equally to the writing of this paper. All authors read and approved the final manuscript.

\section{Author details}

${ }^{1}$ School of Management Science and Engineering, Anhui University of Finance and Economics, Bengbu, 233030, China.

${ }^{2}$ School of Mathematics and Statistics, Hulunbuir College, Hulunbuir, 021008, China.

\section{Publisher's Note}

Springer Nature remains neutral with regard to jurisdictional claims in published maps and institutional affiliations.

Received: 29 June 2017 Accepted: 7 October 2017 Published online: 24 October 2017

\section{References}

1. Sen, M, Banerjee, M, Morozov, A: Bifurcation analysis of a ratio-dependent prey-predator model with the Allee effect. Ecol. Complex. 11, 12-27 (2012)

2. Upadhyay, RK, Agrawal, R: Dynamics and responses of a predator-prey system with competitive interference and time delay. Nonlinear Dyn. 83, 821-837 (2016)

3. Zeng, ZJ: Periodicity in a neutral predator-prey system with monotone functional responses. Adv. Differ. Equ. 2017, Article ID 48 (2017)

4. Yu, SB: Global asymptotic stability of a predator-prey model with modified Leslie-Gower and Holling-type II schemes. Discrete Dyn. Nat. Soc. 2012, Article ID 208167 (2012)

5. Song, YL, Yuan, SL, Zhang, JM: Bifurcation analysis in the delayed Leslie-Gower predator-prey system. Appl. Math. Model. 33, 4049-4061 (2009)

6. Yuan, SL, Song, YL: Stability and Hopf bifurcations in a delayed Leslie-Gower predator-prey system. J. Math. Anal. Appl. 355, 82-100 (2009)

7. Yu, SB: Global stability of a modified Leslie-Gower model with Beddington-DeAngelis functional response. Adv. Differ. Equ. 2014, Article ID 84 (2014)

8. Ghoral, S, Poria, S: Emergent impacts of quadratic mortality on pattern formation in a predator-prey system. Nonlinear Dyn. 87, 2715-2734 (2017)

9. Upadhyay, RK, Tiwari, SK: Ecological chaos and the choice of optimal harvesting policy. J. Math. Anal. Appl. 448 , 1533-1559 (2017)

10. Biswas, S, Sasmal, SK, Samanta, S, Saifuddin, M, Pal, N, Chattopadhyay, J: Optimal harvesting and complex dynamics in a delayed eco-epidemiological model with weak Allee effects. Nonlinear Dyn. 87, 1553-1573 (2017)

11. Xu, R: Global stability and Hopf bifurcation of a predator-prey model with stage structure and delayed predator response. Nonlinear Dyn. 67, 1683-1693 (2012)

12. Ding, $X Q$, Zhao, GF: Periodic solutions for a semi-ratio-dependent predator-prey system with delays on time scales. Discrete Dyn. Nat. Soc. 2012, Article ID 928704 (2012)

13. Zhang, X, Xu, R, Gan, QT: Global stability for a delayed predator-prey system with stage structure for the predator. Discrete Dyn. Nat. Soc. 2009, Article ID 285934 (2009)

14. Zheng, LZ: Stability and Hopf bifurcation of a predator-prey model with distributed delays and competition term Math. Probl. Eng. 2014, Article ID 428523 (2014)

15. Xue, YK, Wang, XQ: Stability and local Hopf bifurcation for a predator-prey model with delay. Discrete Dyn. Nat. Soc. 2012, Article ID 252437 (2012)

16. Upadhyay, RK, Iyengar, SRK: Introduction to Mathematical Modeling an Chaotic Dynamics. CRC Press, Boca Raton (2013) 
17. Hassard, BD, Kazarinoff, ND, Wan, YH: Theory and Applications of Hopf Bifurcation. Cambridge University Press, Cambridge (1981)

18. Bianca, C, Ferrara, M, Guerrini, L: The Cai model with time delay: existence of periodic solutions and asymptotic analysis. Appl. Math. Inf. Sci. 7, 21-27 (2013)

19. Jana, D, Bairagi, N, Agrawal, R, Upadhyay, RK: Modeling the effect of gestation delay of predator on the stability of bifurcating periodic solutions in wetland ecosystem. J. Ecol. 107, 175-189 (2013)

20. Jana, D, Agrawal, R, Upadhyay, RK: Top-predator interference and gestation delay as determinants of the dynamics of a realistic model food chain. Chaos Solitons Fractals 69, 50-63 (2014)

21. Upadhyay, RK, Agrawal, R: Modeling the effect of mutual interference in a delay-induced predator-prey system. J. Appl. Math. Comput. 49, 13-39 (2015)

Submit your manuscript to a SpringerOpen ${ }^{\circ}$ journal and benefit from:

- Convenient online submission

- Rigorous peer review

- Open access: articles freely available online

- High visibility within the field

- Retaining the copyright to your article

Submit your next manuscript at $\gg$ springeropen.com 